\title{
Profile of ramucirumab in the treatment of metastatic non-small-cell lung cancer
}

\author{
This article was published in the following Dove Press journal: \\ OncoTargets and Therapy \\ 4 April 2016 \\ Number of times this article has been viewed
}

\section{Maryann R Cooper ${ }^{1}$ \\ Chelsea Binkowski2,3 \\ Jessica Hartung ${ }^{2,4}$ \\ Jennifer Towle}

'Department of Pharmacy Practice, School of Pharmacy - Worcester/ Manchester, MCPHS University,

Manchester, NH, ${ }^{2}$ School of Pharmacy - Boston, MCPHS University, Boston, MA, ${ }^{3}$ North America Medical Affairs, ${ }^{4}$ Global Medical Affairs, Sanofi

Oncology, Cambridge, MA, USA
Correspondence: Maryann R Cooper School of Pharmacy - Worcester/ Manchester, Massachusetts College of Pharmacy and Health Sciences University, I260 Elm Street, Manchester, $\mathrm{NH} 0310 \mathrm{I}$, USA

$\mathrm{Tel}+\mid 6033141737$

Fax +l 6033140209

Email maryann.cooper@mcphs.edu

\begin{abstract}
The interaction between vascular endothelial growth factor and its receptor is an important therapeutic target due to the importance of this pathway in carcinogenesis. In particular, this pathway promotes and regulates angiogenesis as well as increases endothelial cell proliferation, permeability, and survival. Ramucirumab is a fully human monoclonal antibody that specifically targets the vascular endothelial growth factor receptor-2, the key receptor implicated in angiogenesis. Currently, ramucirumab is approved for the second-line treatment of metastatic non-small-cell lung cancer (NSCLC) in combination with docetaxel. In a Phase III clinical trial, ramucirumab was shown to improve the overall survival in patients with disease progression, despite platinum-based chemotherapy for advanced NSCLC. This review describes the pharmacology, pharmacokinetics and dynamics, adverse event profile, and the clinical activity of ramucirumab observed in Phase II and III trials in NSCLC.
\end{abstract}

Keywords: NSCLC, antiangiogenesis, VEGF-targeted therapy

\section{Introduction}

Angiogenesis, the formation of new blood vessels, is essential for the delivery of oxygen and nutrients to tumor cells and is therefore critical for the growth, invasion, and metastasis of human malignancies. ${ }^{1-3}$ Angiogenesis is a multistep process that is primarily regulated by interactions between vascular endothelial growth factors (VEGFs) and VEGF tyrosine kinase receptors (VEGFRs). ${ }^{1,4,5}$ Of the VEGF family of receptors, VEGFR-2 is considered the key receptor controlling angiogenesis. ${ }^{1,5}$ Specifically, the interaction between VEGF-A and VEGFR-2 promotes and regulates angiogenesis as well as increases endothelial cell proliferation, permeability, and survival.,

VEGF and VEGFR-2 are upregulated in most malignancies, including non-smallcell lung cancer (NSCLC). ${ }^{6}$ Overexpression of VEGF and tumor microvessel density has been associated with increased tumor aggressiveness, an increased risk of recurrence, and a decrease in overall survival (OS) in NSCLC. ${ }^{7-9}$ Therefore, targeting VEGF, in addition to traditional cytotoxic treatments, is a reasonable treatment approach for NSCLC.

Bevacizumab (Avastin ${ }^{\circledR}$; Genentech, Inc., South San Francisco, CA, USA), a fully humanized monoclonal antibody that potently binds VEGF-A preventing its interaction with VEGFR-1 and VEGFR-2, has been shown to improve OS in patients with recurrent or advanced NSCLC. ${ }^{10,11}$ Major hemoptysis occurred more frequently in patients with squamous cell histology; therefore, the drug is recommended only for patients with nonsquamous histologies. ${ }^{10,12}$ Currently, bevacizumab is the only approved anti-VEGF-targeted therapy for first-line treatment of unresectable, locally advanced, recurrent, or metastatic NSCLC in combination with standard chemotherapy. 
Ramucirumab (Cyramza ${ }^{\mathrm{TM}}$, IMC-1121B; Eli Lilly and Company, Indianapolis, IN, USA) offers a unique mechanism of action compared with bevacizumab, in which it binds with high affinity to the extracellular domain of the VEGFR-2 receptor. ${ }^{13,14}$ Ramucirumab has been studied in two Phase II trials as first-line treatment in combination with standard chemotherapy and in a Phase III trial as second-line therapy in combination with docetaxel for patients with advanced or metastatic squamous or nonsquamous NSCLC. ${ }^{15-17}$ This review focuses on the key data and pharmacologic profile of ramucirumab in NSCLC.

\section{Chemistry and mechanism of action} Ramucirumab, $\mathrm{C}_{6376} \mathrm{H}_{9886} \mathrm{~N}_{1702} \mathrm{O}_{1996} \mathrm{~S}_{46}$, is a fully human immunoglobulin G1 monoclonal antibody that inhibits angiogenesis by specifically binding to VEGFR-2. ${ }^{13,14,18}$ The molecular weight of the recombinant compound is $\sim 147 \mathrm{kDa} .{ }^{13,18}$ Two identical heavy chains comprised of 446 amino acids each, and two identical light chains comprised of 214 amino acids each make up the chemical structure of ramucirumab. ${ }^{18}$

Ramucirumab binds with high affinity to the extracellular domain of VEGFR-2, inhibiting the binding of VEGF receptor ligands VEGF-A, VEGF-C, and VEGF-D. ${ }^{13,14}$ Specifically, the half maximal inhibitory concentration for the interaction of VEGF-A and VEGFR-2 is $0.8-1 \mathrm{nM} .{ }^{19}$ The fragment antigen-binding (Fab) region of ramucirumab binds to the end of domain 3 near the $\mathrm{N}$ terminus of the VEGFR-2 receptor. ${ }^{20}$ The binding affinity of ramucirumab to this portion of VEGFR-2 is approximately eight to nine times higher than that of the natural VEGF ligand. ${ }^{21}$ As a result, VEGF-stimulated endothelial cell migration and proliferation are inhibited, ultimately leading to decreased angiogenesis. , $^{3,5,13}$

\section{Pharmacokinetics}

The pharmacokinetic profile of ramucirumab was primarily derived from population data and from two Phase I clinical trials. ${ }^{13,14,22}$ Spratlin et al evaluated weekly 1 hour infusions of ramucirumab with doses escalating from $2-16 \mathrm{mg} / \mathrm{kg}$ in 37 patients with advanced solid tumors. ${ }^{14}$ Chiorean et al investigated ramucirumab 6-10 mg/kg administered every 2 weeks and $12-20 \mathrm{mg} / \mathrm{kg}$ given every 3 weeks in 25 patients with advanced malignancies. ${ }^{22}$ Both Phase I trials found high interpatient variability in the pharmacokinetic parameters. ${ }^{14,22}$

Results of the Spratlin trial revealed that ramucirumab exhibits nonlinear, dose-dependent elimination, with the clearance rate decreasing disproportionately as the dose increased. ${ }^{14}$ This effect was less noticeable with doses
$>8 \mathrm{mg} / \mathrm{kg}$, consistent with saturable clearance, suggesting a receptor-mediated clearance mechanism. Chiorean et al found that the clearance rate of ramucirumab was low and consistent across all doses, with steady state ranging from $0.186-0.255 \mathrm{~mL} / \mathrm{h} / \mathrm{kg}$ across the different dosing levels. ${ }^{22}$ These findings are consistent with the findings of the previous trial, in which most patients received doses $>8 \mathrm{mg} / \mathrm{kg}$. From a population pharmacokinetic analysis, average clearance rate of ramucirumab was found to be $0.015 \mathrm{~L} / \mathrm{h} .{ }^{13}$ The volume of distribution at steady state is $\sim 30-60 \mathrm{~mL} / \mathrm{kg}$, which is similar to that of the total blood volume. ${ }^{22}$

A target trough concentration of $\geq 20 \mu \mathrm{g} / \mathrm{mL}$ was reached following all doses within the Phase I trial conducted by Spratlin et al. ${ }^{14}$ Both trials determined that the terminal half-life is long, ranging from 200-300 hours for doses given weekly and 110-160 hours for doses administered every 2-3 weeks. ${ }^{14,22}$ In addition, Spratlin et al found that the half-life, the maximum concentration $\left(\mathrm{C}_{\max }\right)$, and the area under the concentration-time curve (AUC) increased disproportionately with increasing doses. ${ }^{14}$ In contrast, Chioroen found that the $\mathrm{C}_{\max }$ and $\mathrm{AUC}$ increased as expected with increases in dosage. ${ }^{22}$

Based on the population pharmacokinetic data, age, sex, and race had no significant impact on the pharmacokinetics of ramucirumab. ${ }^{13}$ Renal impairment, as well as mild-tomoderate hepatic impairment, did not significantly alter the concentration at steady state. To date, there has been no formal pharmacokinetic analysis performed in patients with severe hepatic impairment. However, increased encephalopathy, ascites, or hepatorenal syndrome was observed in patients with Child-Pugh $\mathrm{B}$ or $\mathrm{C}$ cirrhosis who received single-agent ramucirumab. No clinically significant drug interactions resulting in changes to the exposure of ramucirumab or any of the approved drug combinations in patients with solid tumors have been reported.

\section{Pharmacodynamics}

Phase I pharmacodynamic studies published to date have investigated the levels of serum VEGF, soluble VEGFR-1 (sVEGFR-1), and soluble VEGFR-2 (sVEGFR-2), in addition to posttreatment changes in tumor perfusion and vascularity using dynamic contrast-enhanced magnetic resonance imaging parameters. ${ }^{14,22}$

Ramucirumab has a higher binding affinity for the extracellular domain of VEGFR-2 than the natural VEGF-A ligand; therefore, following treatment, VEGF-A concentrations increased and remained elevated for at least 7 days posttreatment in both Phase I trials. ${ }^{14,22}$ VEGF-A levels rose 
1.5-3.5 times higher than pretreatment levels in the Spratlin trial and 2-8 times higher in the Chiorean study. The rise in VEGF-A levels was independent of the ramucirumab dose. In contrast, sVEGFR-2 concentrations decreased following treatment with ramicurumab in both trials. sVEGFR-1 decreased following treatment in the Spratlin trial and remained constant in the Chiorean study.

Dynamic contrast-enhanced magnetic resonance imaging parameters evaluated in both Phase I trials included initial area under the signal-intensity curve, volume of extravascular extracellular space, and uptake rate constant. ${ }^{14,22}$ Results demonstrated a decline in these parameters indicating decreased tumor perfusion and vascularity and an antiangiogenic response in most evaluable patients (nine out of 13 [69\%] and four out of nine [67\%], respectively).

\section{Immunogenicity}

In the Phase I trial conducted by Spratlin et al, antiramucirumab antibodies were not detected in 26 patients. ${ }^{14}$ Three out of $25(12 \%)$ patients evaluated pre- and posttreatment in the trial conducted by Chiorean et al developed antiramucirumab antibodies. ${ }^{22}$ These antibodies were detected in two patients before the second cycle of the 15 and $20 \mathrm{mg} / \mathrm{kg}$ infusions given every 3 weeks. The remaining patient had antibodies discovered at follow-up after receiving $6 \mathrm{mg} / \mathrm{kg}$ every 2 weeks. Of note, none of the antibodies detected in these patients were neutralizing.

\section{Clinical trials Phase II trials}

To date, ramucirumab has been studied as a first-line treatment option in two open-label Phase II studies. ${ }^{15,16}$ Data from these trials and the Phase III trial are summarized in Table 1. The first study was a single-arm, multicenter trial designed to evaluate the safety and efficacy of ramucirumab $10 \mathrm{mg} / \mathrm{kg}$ given intravenously (IV) every 21 days in combination with paclitaxel $\left(200 \mathrm{mg} / \mathrm{m}^{2}\right)$ and carboplatin (AUC 6). ${ }^{15}$ Patients were included if they were at least 18 years of age, had untreated stage IIIB/IV NSCLC with an Eastern Cooperative Oncology Group performance status (ECOG PS) of 0-1, and adequate renal and hepatic function.

The primary end point of this study was the 6-month progression free survival (PFS) rate with secondary end points being PFS, OS, objective response rate (ORR), safety/

Table I Summary of data on completed clinical trials of ramucirumab

\begin{tabular}{|c|c|c|c|c|}
\hline Reference & Design & Regimen & $\begin{array}{l}\text { Patient } \\
\text { population }\end{array}$ & End points ${ }^{\mathrm{a}, \mathrm{b}}$ \\
\hline $\begin{array}{l}\text { Camidge } \\
\text { et } \mathrm{al}^{15}\end{array}$ & $\begin{array}{l}\text { Phase II, open-label, } \\
\text { single arm, and } \\
\text { multicenter }\end{array}$ & $\begin{array}{l}\text { Ramucirumab with } \\
\text { paxlitaxel + carboplatin } \\
(n=40)\end{array}$ & $\begin{array}{l}\text { Adult patients } \\
\text { with stage IIIB } \\
\text { or IV NSCLC }\end{array}$ & $\begin{array}{l}\text { Six-month PFS rate: } 59 \%(95 \% \mathrm{Cl}, 4 \mathrm{I} .3 \%-72.9 \%) \\
\text { Median PFS: } 7.85 \mathrm{mo}(95 \% \mathrm{Cl}, 5.49-9.86 \mathrm{mo}) \\
\text { Median OS: } 16.85 \mathrm{mo}(95 \% \mathrm{Cl}, 14.82-28.58 \mathrm{mo}) \\
\text { ORR: } 55 \%(95 \% \mathrm{Cl}, 38.5 \%-70.7 \%) \\
\text { DCR: } 90.0 \%(95 \% \mathrm{Cl}, 76.3 \%-97.2 \%)\end{array}$ \\
\hline $\begin{array}{l}\text { Doebele } \\
\text { et } \text { al }^{16}\end{array}$ & $\begin{array}{l}\text { Phase II, open-label, } \\
\text { randomized control } \\
\text { trial, and international } \\
\text { multicenter }\end{array}$ & $\begin{array}{l}\text { Ramucirumab with } \\
\text { pemetrexed + carboplatin } \\
\text { or cisplatin }(n=67) \text { vs } \\
\text { pemetrexed + carboplatin } \\
\text { or cisplatin }(n=69)\end{array}$ & $\begin{array}{l}\text { Adult patients } \\
\text { with stage IV } \\
\text { nonsquamous } \\
\text { NSCLC }\end{array}$ & $\begin{array}{l}\text { Median PFS: } 7.2 \mathrm{mo}(90 \% \mathrm{Cl}, 5.8-8.4 \mathrm{mo}) \text { vs } \\
5.6 \mathrm{mo}(90 \% \mathrm{Cl}, 4.0-5.7 \mathrm{mo}) \\
\text { Median OS: I3.9 mo }(90 \% \mathrm{Cl}, 10.0-17.8 \mathrm{mo}) \text { vs } \\
\text { I0.4 mo }(90 \% \mathrm{Cl}, 8.2-15.9 \mathrm{mo}) \\
\text { ORR: } 49.3 \%(90 \% \mathrm{Cl}, 39.4 \%-59.2 \%) \text { vs } \\
38 \%(90 \% \mathrm{Cl}, 28.6 \%-47.5 \%) \\
\text { DCR: } 85.5(90 \% \mathrm{Cl}, 78.5 \%-92.5 \%) \text { vs } \\
70.4 \%(90 \% \mathrm{Cl}, 6 \mathrm{I} .5 \%-79.3 \%) P=0.032\end{array}$ \\
\hline $\begin{array}{l}\text { Garon } \\
\text { et } \mathrm{al}^{17}\end{array}$ & $\begin{array}{l}\text { Phase III, multicenter, } \\
\text { international double } \\
\text { blind, randomized, } \\
\text { and placebo- } \\
\text { controlled trial }\end{array}$ & $\begin{array}{l}\text { Ramucirumab with } \\
\text { docetaxel }(n=628) \text { vs } \\
\text { placebo with docetaxel } \\
(n=625)\end{array}$ & $\begin{array}{l}\text { Adult patients } \\
\text { with stage IV } \\
\text { NSCLC }\end{array}$ & $\begin{array}{l}\text { Median OS: I0.5 mo }(95 \% \mathrm{Cl}, 9.5-\mathrm{II} .2 \mathrm{mo}) \text { vs } \\
\text { 9.I mo (95\% Cl, 8.4-I0.0 mo) } \\
\text { HR } 0.86(95 \% \mathrm{Cl}, 0.75-0.98 ; P=0.023) \\
\text { Median PFS: } 4.5 \mathrm{mo}(95 \% \mathrm{Cl}, 4.2-5.4 \mathrm{mo}) \text { vs } \\
3.0 \text { mo (95\% Cl, 2.8-3.9 mo) } \\
\text { HR } 0.76(95 \% \mathrm{Cl}, 0.68-0.86 ; P<0.000 \mathrm{I}) \\
\text { ORR: } 23 \% \text { vs I4\% } \\
\text { OR I.89 (95\% Cl, I.4I-2.54; } P<0.000 \mathrm{I}) \\
\text { DCR } 64 \% \text { vs } 53 \% \\
\text { OR I.60 (95\% Cl, I.28-2.0I; } P<0.000 \mathrm{I})\end{array}$ \\
\hline
\end{tabular}

Notes: aPrimary end points. 'When applicable, outcomes are for ramucirumab vs comparator regimen, respectively. Copyright @ 2015 . Wiley and Sons. Adapted from Doebele RC, Spigel D, Tehfe M, et al. Phase 2, randomized, open-label study of ramucirumab in combination with first-line pemetrexed and platinum chemotherapy in patients with nonsquamous, advanced/metastatic non-small cell lung cancer. Cancer. 20I5; I2I(6):883-892.16

Abbreviations: $\mathrm{Cl}$, confidence interval; DCR, disease control rate; HR, hazard ratio; mo, months; NSCLC, non-small-cell lung cancer; OS, overall survival; OR, odds ratio; ORR, objective response rate; PFS, progression free survival. 
tolerability, pharmacokinetic parameters, and potential biomarkers correlated with clinical outcomes. ${ }^{15}$

Baseline characteristics of the 40 patients enrolled revealed a median age of 59.5 years (range: $35-78$ years), mostly non-Hispanic Caucasian (92.5\%) females (62.5\%) with stage IV NSCLC (82.5\%) with adenocarcinoma histology $(85.0 \%) .{ }^{15}$ At 6 months, the PFS rate was $59.0 \%(95 \%$ confidence interval [CI], 41.3\%-72.9\%) with 15 patients (37.5\%) experiencing disease progression during the first 6 months of therapy. By the end of the 3-year data collection period, the median PFS was 7.85 months $(95 \%$ CI, 5.49-9.86 months) and the median OS was 16.85 months (95\% CI, 14.82-28.58 months). At the time of data cutoff, 14 patients $(35.0 \%)$ were still alive. One patient $(2.5 \%)$ experienced a complete response (CR) and 21 patients (52.5\%) experienced a partial response (PR) making the ORR (CR + PR) 55.0\% (95\% CI, 38.5\%-70.7\%) and the disease control rate (DCR [CR + PR + stable disease]) $90.0 \%(95 \%$ CI, 76.3\%-97.2\%).

Adverse drug reactions (ADRs) were experienced by 34 patients $(85.0 \%)$ (Table 2$).{ }^{15}$ The majority of ADRs reported were Common Terminology Criteria for Adverse Events (CTCAE) grade 2 or 3 . The most common reactions of any grade included fatigue, neuropathy/myalgi, nausea, and epistaxis. Grade 3 adverse events emerged in ten patients $(25.0 \%)$ and included neutropenia/febrile neutropenia, thrombocytopenia, fatigue, peripheral neuropathy, anemia, constipation, anorexia, dyspnea, and hypertension. A total of five patients (12.5\%) experienced a grade 4 ADR, including febrile neutropenia, pulmonary embolism, thrombocytopenia, and neutropenia. Thirteen patients $(32.5 \%)$ discontinued treatment due to ADRs. The most common ADRs leading to discontinuation occurred within the first six cycles and included infusion-related reactions (not attributed to ramucirumab), neutropenia, and fatigue.

Biomarkers were analyzed in 22 of the non-Hispanic Caucasian participants. ${ }^{15}$ This analysis revealed that the single-nucleotide polymorphism rs2981582 on the FGFR-2 gene was statistically significantly associated with $\mathrm{OS}(P=0.0059)$, PFS $(P=0.0429)$, and best overall response rate $(P=0.0392)$. In addition, high serum $I L-6$ levels were associated with decreases in PFS $(P=0.0038)$ and $\mathrm{OS}(P=0.0029)$.

The second Phase II trial was a randomized, open-label, and international study evaluating the safety and efficacy of ramucirumab $10 \mathrm{mg} / \mathrm{kg}$ IV every 21 days given in combination with pemetrexed $\left(500 \mathrm{mg} / \mathrm{m}^{2}\right)$ and platinum chemotherapy (carboplatin AUC 6 or cisplatin $75 \mathrm{mg} / \mathrm{m}^{2}$ ) $(n=67)$ compared with pemetrexed and platinum therapy alone $(n=69) .{ }^{16}$ Patients selected for this study were at least 18 years of age with previously untreated stage IV nonsquamous NSCLC and an ECOG PS of 0-2. The study was designed to identify the primary outcome of PFS, with $60 \%$ power. Secondary end points included OS, ORR, DCR, and safety.

Baseline characteristics between the groups were similar except that the ramucirumab arm had a greater percentage of female patients compared with the control group $(47.8 \%$ vs $36.6 \%$, respectively). ${ }^{16}$ The patient population was composed of mostly nonsmoking $(77.5 \%$ ramucirumab and $84.1 \%$ control), Caucasians (91.5\% ramucirumab and $87 \%$ control), with ECOG PS of 0-1 (91.5\% ramucirumab and $92.8 \%$ control), and adenocarcinoma (87.3\% ramucirumab and $87 \%$ control). The median PFS for the ramucirumab arm was 7.2 months (90\% CI, 5.8-8.4 months) compared with 5.6 months (90\% CI, 4.0-5.7 months) in the control group. As established by the hazard ratio (HR), this difference was not statistically significant (HR 0.75 [90\% CI, 0.55-1.03; $P=0.132]$ ). Median OS was prolonged by 3.5 months in the ramucirumab group compared with the control group (13.9 months [90\% CI, 10.0-17.8 months] vs 10.4 months [90\% CI, 8.2-15.9 months], respectively). However, this result was not statistically different (HR 1.03 [90\% CI, $0.74-1.42 ; P=0.892])$. One patient $(1.4 \%)$ experienced a $\mathrm{CR}$, $33(47.8 \%)$ had a PR, and $25(36.2 \%)$ had stable disease in the ramucirumab group compared with no CRs, 27 (38.0\%) PRs, and 23 (32.4\%) with stable disease in the control group. This translated into a statistically significant difference in the DCR (85.5\% [90\% CI, 78.5\%-92.5\%] ramucirumab vs $70.4 \%$ [90\% CI, 61.5\%-79.3\%] control, $P=0.032$ ). However, the ORR of $49 \%(90 \%$ CI, 39.4\%-59.2\%) in the ramucirumab group and $38 \%(90 \%$ CI, $28.6 \%-47.5 \%)$ in the control group was not statistically different $(P=0.180)$.

The number of patients experiencing an ADR of any CTCAE grade was similar between the two groups (Table 2). ${ }^{16}$ More patients discontinued at least one component of treatment due to an ADR in the ramucirumab group $(n=22[32.8 \%])$ compared with the control group $(n=14$ [20.3\%]). Grade 3 thrombocytopenia led to discontinuation in three $(4.5 \%)$ of the ramucirumab patients. No particular ADR was listed for the remaining patients. The most common ( $\geq 20 \%$ ) ADRs of any grade reported more frequently in the ramucirumab arm included fatigue, thrombocytopenia, neutropenia, anorexia, peripheral edema, headache, epistaxis, back pain, insomnia, and dyspnea. Of the grade 3 ADRs that occurred in $\geq 10 \%$ of patients, thrombocytopenia, neutropenia, nausea, and hypertension occurred more frequently in 


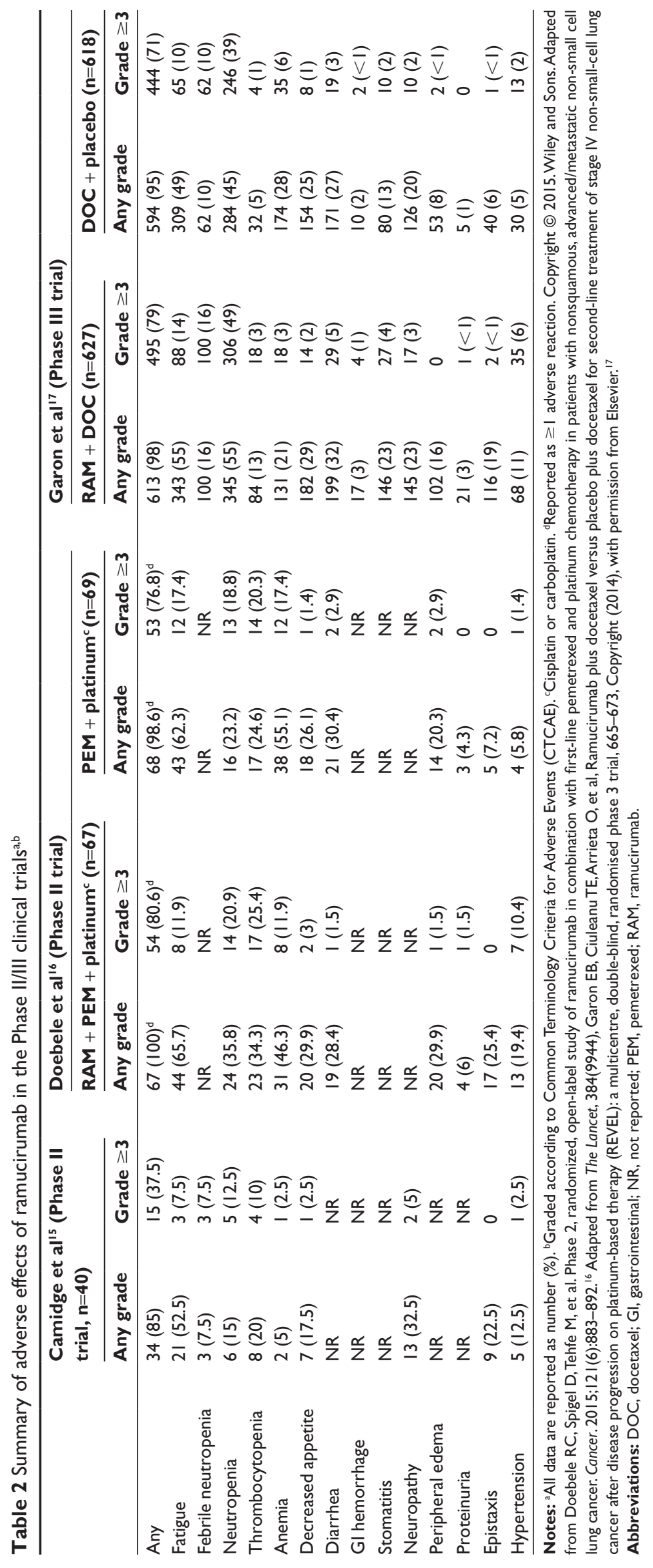


the ramucirumab group compared with the control group. Eight grade 5 events occurred during the trial, three (4.5\%) in the ramucirumab arm (myocardial infarction, multiorgan failure, and sudden death) and five (7.2\%) in the control arm (cardiorespiratory arrest, embolism, hemothorax, myocardial infarction, and respiratory failure).

\section{Phase III}

The REVEL trial was a Phase III, multicentered, randomized, double-blind, and placebo-controlled trial designed to compare ramucirumab $10 \mathrm{mg} / \mathrm{kg}$ IV every 21 days plus docetaxel $\left(75 \mathrm{mg} / \mathrm{m}^{2}\right)$ with docetaxel plus placebo in patients with stage IV NSCLC. ${ }^{17,23}$ To be included, patients had to be at least 18 years of age, have an ECOG PS of $0-1$, stage IV disease with progression during or after one first-line platinum-based regimen, and adequate organ function. Patients who had received previous treatment with bevacizumab were eligible for this study, which is a distinction in the previous Phase II trials in which patients receiving this agent were excluded.

The primary outcome of this trial was OS with secondary end points of PFS, ORR, DCR, safety, and quality of life (QOL). ${ }^{17}$ In addition, the immunogenicity of ramucirumab and potential predictive biomarkers were also investigated. Investigation of these areas is still ongoing.

Baseline characteristics between the two groups were similar. ${ }^{17}$ The median age was 62 years (range: $21-85$ years) in the ramucirumab group $(n=628)$ and 61 years (range: $25-86$ years $)$ in the control group $(n=625)$. Most patients were Caucasian (84\% ramucirumab and $80 \%$ control), males $(67 \%$ ramucirumab and $66 \%$ control), with a previous history of smoking ( $82 \%$ ramucirumab and $77 \%$ control), an ECOG PS of 1 (67\% ramucirumab and $68 \%$ control), and nonsquamous histology (74\% ramucirumab and 72\% control).

Median OS was 10.5 months (95\% CI, 9.5-11.2 months) compared with 9.1 months (95\% CI, 8.4-10.0 months) in the control group. ${ }^{17}$ This difference was statistically significant with a HR of $0.86(95 \% \mathrm{CI}, 0.75-0.98 ; P=0.023)$. A trend toward improved OS was observed in the nonsquamous histology (HR 0.83 [95\% CI, 0.71-0.97]), squamous cell histology (HR 0.88 [95\% CI, 0.69-1.13]), and patients who responded to the first-line platinum therapy subgroups (HR $0.84[95 \%$ CI, 0.71-0.99]). Patients deemed nonresponders to first-line platinum therapy had similar OS between the two arms. Median PFS also significantly increased in the ramucirumab group (4.5 months [95\% CI, 4.2-5.4 months]) compared with the control group (3.0 months [95\% CI, 2.8-3.9 months]), with an HR of 0.76 (95\% CI, 0.68-0.86; $P<0.0001)$. These results were sustained across subgroups after accounting for baseline characteristics, including squamous versus nonsquamous histology. A total of three patients $(0.5 \%)$ had a CR and 144 patients (22.5\%) experienced a PR in the ramucirumab group as compared with two $(0.3 \%) \mathrm{CR}$ and $83(13.3 \%)$ PR in the control group. Thus, the ORR was higher in the ramucirumab group (23\%) as compared with the control group (14\%) (odds ratio [OR] 1.89 [95\% CI, 1.41-2.54; $P<0.0001])$. DCR was also statistically significantly higher with ramucirumab (64\%) compared with the control group (53\%) (OR 1.60 [95\% CI, 1.28-2.01; P<0.0001]).

Most patients experienced at least one ADR of any CTCAE grade (Table 2). ${ }^{17}$ Dose adjustments were made in a higher percentage of ramucirumab patients $(n=204$ [33\%]) compared with control patients $(n=139$ [23\%]). The most common ADRs resulting in dose adjustments were neutropenia ( $\mathrm{n}=77[12 \%]$ ramucirumab vs $\mathrm{n}=55$ [9\%] control), fatigue $(n=54[9 \%]$ ramucirumab vs $n=34[6 \%]$ control), and febrile neutropenia $(n=44[7 \%]$ ramucirumab vs $n=28$ [5\%] controls). Although the rate of febrile neutropenia was higher in the ramucirumab group, the use of growth factor support was similar. Bleeding and hemorrhage occurred more frequently in the ramucirumab arm $(n=181$ [29\%] ramucirumab vs $n=94$ [15\%] control); however, the incidence of grade 3 or higher bleeding was similar between the two groups. The incidence of hypertension was higher in the ramucirumab arm $(n=68$ [11\%] ramucirumab vs $n=30$ [5\%] control) with one account of grade 4 elevation. Approximately half of the patients in each arm completed the QOL analysis, which showed there was no statistical difference between the two arms (stratified HR 1.00 [95\% CI, 0.84-1.19; $P=0.99]$ ).

\section{Ongoing trials}

There are currently three Phase I, one Phase II, and one Phase III trial in progress, evaluating the effects of ramucirumab in patients with advanced NSCLC (Table 3). The NCT02443324 Phase I trial is a multicenter, international, and open-label trial set to evaluate the safety outcomes of ramucirumab in combination with pembrolizumab in different types of advanced cancers, including nonsquamous and squamous NSCLC. The primary end point is dose-limiting toxicities with secondary end points of CR, PR, ORR, DCR, duration of response, PFS, time to first response, OS, and pharmacokinetic parameters.

The NCT02079636 Phase I trial is an open-label, multicenter, and dose escalation study, evaluating the combination of test drug LY2835219 with various anticancer medications in advanced NSCLC patients. One arm of the trial will compare the test drug with ramucirumab, while other 
Table 3 Ongoing clinical trials of ramucirumab in NSCLC

\begin{tabular}{|c|c|c|}
\hline Trial & Phase & Identifier \\
\hline Multicenter, open-label ramucirumab plus pembrolizumab for advanced unrespectable cancers & Phase I & NCT02443324 \\
\hline Open-label, dose escalation trial of LY2835219 combination therapy for stage IV NSCLC & Phase I & NCT02079636 \\
\hline Open-label, dose escalation trial of LY2875358, and ramucirumab combination therapy in advanced cancer & Phase I+II & NCT02082210 \\
\hline $\begin{array}{l}\text { Multicenter, randomized, double-blind erlotinib plus ramucirumab vs erlotinib plus placebo in the treatment } \\
\text { of EGFR mutation-positive metastatic NSCLC }\end{array}$ & Phase III & NCT024II448 \\
\hline
\end{tabular}

Abbreviations: EGFR, epidermal growth factor receptor; NSCLC, non-small-cell lung cancer.

arms in this trial will evaluate the test drug in combination with gemcitabine, pemetrexed, or LY3023414. Primary end points include drug-related ADRs and secondary end points include CR, PR, ORR, pharmacokinetic parameters, PFS, and change from baseline on the MD Anderson Symptom Inventory Scale-Lung Cancer.

The NCT02082210 trial has two phases planned. Part 1 will include a dose escalation phase, evaluating the combination of LY2875358 and ramucirumab with the primary end point of dose-limiting toxicities. The planned extension of this trial will evaluate the safety and tumor response using CR, PR, and ORR. Secondary end points for both phases will include pharmacokinetic parameters, DCR, stable disease, and PFS.

The NCT02411448 trial is a randomized, double-blind, and multicenter study comparing ramucirumab plus erlotinib versus erlotinib plus placebo in patients with EGFR mutationpositive metastatic NSCLC. The primary end points include PFS and drug-related ADRs. Secondary end points include OS, ORR, DCR, duration of response, pharmacokinetic parameters, antiramucirumab antibodies, and changes from baseline in the Lung Cancer Symptom Scale and the EuroQol 5-Dimension, 5-Level Questionnaire (EQ-5D-5L).

\section{Conclusion}

Agents that target the VEGF pathway represent an important pharmacologic class for the treatment of malignancy due to the importance of this pathway in the process of angiogenesis and cell proliferation. Ramucirumab offers a unique mechanism of action in that it specifically targets the VEGFR-2 receptor, the key receptor thought to drive angiogenesis. Currently, ramucirumab is approved for the second-line treatment of metastatic NSCLC in combination with docetaxel. In a Phase III clinical trial, ramucirumab was shown to improve OS in patients with disease progression, despite platinum-based chemotherapy for advanced NSCLC. This improvement in OS is encouraging; however, many questions remain regarding the utility of this agent.
One question left unanswered is the optimal sequence of this agent in the treatment of NSCLC. Although it is approved as a second-line therapy and improves OS in this setting, there was an improvement in DCR seen in the first-line setting in patients with nonsquamous histology in a Phase II trial. Given the potential for clinical benefit in the first-line arena, further investigation is warranted. At this time, it is unclear whether the drug combination with ramucirumab leads to improved OS in the first-line setting and whether this improvement could be compared with that seen with bevacizumab. In addition, a comparison of ramucirumab with other VEGF-targeted therapies, bevacizumab in particular, would be useful to determine the target of this agent in the treatment of NSCLC.

It is also important to consider the generalizability of the published data. In the current clinical trials, the average age of participants was $\sim 60$ years, whereas the average age at the diagnosis of NSCLC is 70 years of age. In addition, the majority of patients studied had an ECOG PS of 0-1; therefore, the safety and efficacy of ramucirumab in more functionally impaired patients are not known.

Another unanswered question is whether there are measurable predictive factors reliably associated with response to ramucirumab. In one Phase II trial, single-nucleotide polymorphism rs2981582 on the FGFR-2 gene was statistically significantly associated with improvements in OS, PFS, and best overall response rate. These data, however, were only collected from a small portion of the study population. Biomarker analysis in the Phase III trial is still ongoing. The lack of validated predictive biomarkers restricts the ability to tailor ramucirumab to specific patient cohorts and remains a barrier to the success of individualized NSCLC therapy. Nevertheless, predictive biomarker research of angiogenesis inhibitors is a growing field and is currently under exploration with ramucirumab.

Furthermore, data pertaining to the complete impact of ramucirumab on QOL are lacking. In the pivotal Phase III trial, QOL was studied as a secondary end point, but it was only assessed in half of the study participants. Although 
no significant difference was observed, this may be due to inefficient power. Another important query is the clinical significance of the OS benefit observed with ramucirumab in this trial. Although there were rather minimal added side effects for patients in the ramucirumab arm, ultimately, the median OS improved by only $\sim 5$ weeks compared with standard chemotherapy alone. This statistically significant yet marginally absolute survival benefit may cause some reservations about its clinical meaningfulness. Finally, formal pharmacoeconomic evaluations need to be completed to ascertain whether the costs associated with the administration of ramucirumab justify the benefits.

\section{Disclosure}

Chelsea Binkowski and Jessica Hartung are employed by Sanofi Oncology. This company does not produce any competing products for the treatment of lung cancer. The other authors report no conflicts of interest in this work.

\section{References}

1. Klagsbrun M, D'Amore PA. Vascular endothelial growth factor and its receptors. Cytokine Growth Factor Rev. 1996;7(3):259-270.

2. Hsu JY, Wakelee HA. Monoclonal antibodies targeting vascular endothelial growth factor: current status and future challenges in cancer therapy. BioDrugs. 2009;23(5):289-304.

3. Aprile G, Bonotto M, Ongaro E, Pozzo C, Giuliani F. Critical appraisal of ramucirumab (IMC-1121B) for cancer treatment: from benchside to clinical use. Drugs. 2013;73(18):2003-2015.

4. Ferrara N. Binding to the extracellular matrix and proteolytic processing: two key mechanisms regulating vascular endothelial growth factor action. Mol Biol Cell. 2010;21(5):687-690.

5. Clarke JM, Hurwitz HI. Targeted inhibition of VEGF receptor 2: an update on ramucirumab. Expert Opin Biol Ther. 2013;13(8):1187-1196.

6. Ferrara N. Vascular endothelial growth factor as a target for anticancer therapy. Oncologist. 2004;9(suppl 1):2-10.

7. Han H, Silverman J, Santucci T. Vascular endothelial growth factor expression in stage I non-small cell lung cancer correlates with neoangiogenesis and a poor prognosis. Ann Surg Oncol. 2001;8:72-79.

8. Mineo TC, Ambrogi V, Baldi A, et al. Prognostic impact of VEGF, CD31, $\mathrm{CD} 34$, and $\mathrm{CD} 105$ expression and tumour vessel invasion after radical surgery for IB-IIA non-small cell lung cancer. J Clin Pathol. 2004;57(6): 591-597.

9. Bremnes RM, Camps C, Sirera R. Angiogenesis in non-small cell lung cancer: the prognostic impact of neoangiogenesis and the cytokines VEGF and bFGF in tumours and blood. Lung Cancer. 2006;51(2): $143-158$.

OncoTargets and Therapy

\section{Publish your work in this journal}

OncoTargets and Therapy is an international, peer-reviewed, open access journal focusing on the pathological basis of all cancers, potential targets for therapy and treatment protocols employed to improve the management of cancer patients. The journal also focuses on the impact of management programs and new therapeutic agents and protocols on

Submit your manuscript here: http://www.dovepress.com/oncotargets-and-therapy-journal
10. Johnson DH, Fehrenbacher L, Novotny WF, et al. Randomized phase II trial comparing bevacizumab plus carboplatin and paclitaxel with carboplatin and paclitaxel alone in previously untreated locally advanced or metastatic non-small-cell lung cancer. J Clin Oncol. 2004;22(11): 2184-2191.

11. Sandler A, Gray R, Perry MC, et al. Paclitaxel-carboplatin alone or with bevacizumab for non-small-cell lung cancer. N Engl J Med. 2006; 355(24):2542-2550.

12. Avastin (bevacizumab) [package insert]. South San Fransisco, CA: Genentech, Inc.; 2015.

13. Cyramza (ramucirumab) [package insert]. Indianapolis, IN: Eli Lilly and Company; 2015.

14. Spratlin J, Cohen R, Eadens M, et al. Phase I pharmacologic and biologic study of ramucirumab (IMC-1121B), a fully human immunoglobulin G1 monoclonal antibody targeting the vascular endothelial growth factor receptor-2. J Clin Oncol. 2010;28(5):780-787.

15. Camidge DR, Berge EM, Doebele RC, et al. A phase II, open-label study of ramucirumab in combination with paclitaxel and carboplatin as first-line therapy in patients with stage IIIB/IV non-small-cell lung cancer. J Thorac Oncol. 2014;9(10):1532-1539.

16. Doebele RC, Spigel D, Tehfe M, et al. Phase 2, randomized, openlabel study of ramucirumab in combination with first-line pemetrexed and platinum chemotherapy in patients with nonsquamous, advanced/ metastatic non-small cell lung cancer. Cancer. 2015;121(6):883-892.

17. Garon EB, Ciuleanu TE, Arrieta O, et al. Ramucirumab plus docetaxel versus placebo plus docetaxel for second-line treatment of stage IV non-small-cell lung cancer after disease progression on platinum-based therapy (REVEL): a multicentre, double-blind, randomised phase 3 trial. Lancet. 2014;384(9944):665-673.

18. Wadhwa R, Taketa T, Sudo K, Blum-Murphy M, Ajani JA. Ramucirumab: a novel antiangiogenic agent. Future Oncol. 2013;9(6):789-795.

19. Spratlin JL, Mulder KE, Mackey JR. Ramucirumab (IMC-1121B): a novel attack on angiogenesis. Future Oncol. 2010;6(7):1085-1094.

20. Franklin MC, Navarro EC, Wang Y, et al. The structural basis for the function of two anti-VEGF receptor 2 antibodies. Structure. 2011;19(8): 1097-1107.

21. Miao HQ, Hu K, Jimenez X, et al. Potent neutralization of VEGF biological activities with a fully human antibody fab fragment directed against VEGF receptor 2. Biochem Biophys Res Commun. 2006;345(1): 438-445.

22. Chiorean EG, Hurwitz HI, Cohen RB, et al. Phase I study of every 2- or 3 -week dosing of ramucirumab, a human immunoglobulin G1 monoclonal antibody targeting the vascular endothelial growth factor receptor-2 in patients with advanced solid tumors. Ann Oncol. 2015;26(6): $1230-1237$.

23. Garon EB, Cao D, Alexandris E, John WJ, Yurasov S, Perol M. A randomized, double-blind, phase III study of docetaxel and ramucirumab versus docetaxel and placebo in the treatment of stage IV non-small-cell lung cancer after disease progression after 1 previous platinum-based therapy (REVEL): treatment rationale and study design. Clin Lung Cancer. 2012;13(6):505-509.

\section{Dovepress}

patient perspectives such as quality of life, adherence and satisfaction. The manuscript management system is completely online and includes a very quick and fair peer-review system, which is all easy to use. Visit http://www.dovepress.com/testimonials.php to read real quotes from published authors. 